\title{
CARACTERIZACIÓN DE LAS DIFERENTES MANERAS DE CONCEBIR LA EXPERIMENTACIÓN EN EL AULA ENTRE DOCENTES DEL GRADO 6 Y 7 DEL INSTITUTO PEDAGÓGICO NACIONAL (I.P.N USAQUÉN, BOGOTÁ D.C ${ }^{1}$ )
}

\section{CHARACTERIZATION OF THE DIFFERENT WAYS THAT INSITUTO PEDAGOGICO NACIONAL TEACHERS OF SIXTH TO NINTH GRADE CONCEIVE EXPERIMENTATION IN THE CLASSROOM}

\begin{tabular}{|l|}
\hline Recibido:09-09-2011 \\
\hline Aceptado:18-12-2011 \\
\hline
\end{tabular}

\section{Por: Fabián Bautista, Johanna Cárdenas, Andrés Castiblanco².}

\section{Resumen}

Este trabajo presenta los resultados y análisis obtenidos a partir del desarrollo del proyecto semestral llevado a cabo desde el eje curricular Diversidad, entendido éste como el proyecto de formación de maestros de tercer semestre de Licenciatura en Biología en la Universidad Pedagógica Nacional. Desde lo anterior el texto tiene como fin la caracterización de las diferentes maneras de concebir la experimentación en el aula entre los profesores del grado sexto y séptimo del Instituto Pedagógico Nacional (I.P.N) para lo cual se propuso un reconocimiento, análisis y reflexión de las mismas dentro del contexto escolar.

El trabajo se realizó bajo el enfoque cualitativo, a partir de dos momentos durante el trascurso del semestre académico que se comprendió entre los meses de Agosto y Noviembre del 2010: En primera instancia, visitas a los espacios de clase como ética y valores, Sociales entre otros y la aplicación de herramientas para la recolección de información, entre ellas la entrevista semiestructurada aplicada a 8 profesores de diferentes áreas como historia, geografía, biología, ingles, lengua castellana y educación física, a partir de lo cual se observaron las estrategias usadas por los docentes con referencia al abordaje de cada una de las temáticas.

Como resultado se obtuvo que los profesores conciben la experimentación en primer lugar como el encuentro de los estudiantes con la experiencia física, los trabajos manuales, los juegos didácticos, que le posibiliten al estudiante adoptar el tema de manera sencilla, y lo aproxime a un contexto socio-cultural, esto lo propenden mediante los recursos que proporciona la institución educativa.

\footnotetext{
${ }^{1}$ Investigación desarrollada a través de metodología cualitativa. Llevada a cabo en el periodo académico 2010-II (Agosto - Diciembre) correspondiente al Eje Curricular Diversidad (3er semestre) de Licenciatura en Biología. Universidad Pedagógica Nacional. No tuvo financiación.

${ }^{2}$ Estudiantes de Licenciatura en Biología de la Universidad Pedagógica Nacional. Contáctese a: dbi fcastiblanco564@pedagogica.edu.co
} 


\section{Abstract}

This article presents the results and analysis obtained from semi-annual project development carried out from the curricular axis, Diversity, which is a project of teachers training of third semester who study Biology education degree at Universidad Pedagógica Nacional. From the above, this paper aims the characterization of different ways of conceiving experimentation in the classroom among teachers of sixth and seventh grade of Instituto Pedagógico Nacional (Usaquén, Bogota D.C). For this a for recognition, analysis and reflection of these within the school context was proposed.

The article was carried out under the qualitative approach, from two moments in the course of the semester, from August to November. First of all, visits to classes such as ethics and values, social sciences and others and application of tools for data gathering, including semi-structured interviews applied to 8 teachers from different subjects such as History, Geography, Biology, English, Spanish and physical education, from which we observed the strategies used by teachers with regard to addressing each theme.

The result was that teachers conceive experimentation in the first place as the meeting that students have with physical experience, crafts, didactic games, which will enable the student to tackle the topic in a simple way, and approach him to a socio-cultural context, this will tend by the resources provided by the school.

Key Words: Conceptions, teacher, experimentation.

\section{Introducción:}

Referirse a la experimentación desde el aula implica un dialogo abierto y permanente del maestro con la realidad porque es a través de esta que se pueden facilitar procesos de aprendizaje vinculados con la experiencia física (Piaget, 1981). Como lo mencionan Marismas de Odiel y Francisco Pozuelos en el seminario de formación en centro "Proyecto escuelas que investiga "La experimentación en el aula constituye uno de los referentes más valiosos a la hora de mejorar la actividad educativa pues conecta de manera fluida los aspectos teóricos y la dimensión práctica de la enseñanza. Con esta interacción se favorece un conocimiento profesional bastante versátil y significativo capaz de ser utilizado de diferente manera y en múltiples situaciones gracias a la implicación sustantiva de los participantes que se alejan de este modo tanto de la adquisición de rutinarias técnicas (recetas) de limitada funcionalidad como de elaborados discursos (eslóganes) de escaso calado práctico."

Desde el enfoque del presente trabajo se busca caracterizar las diferentes maneras de concebir la experimentación en el aula entre los profesores de los grados 6 y 7 del IPN (Usaquén, Bogotá), partiendo de su reconocimiento, análisis y reflexión de las mismas dentro del contexto escolar. Lo anterior toma importancia pues a partir del cuestionamiento de nuestro propio proceso de formación se ve posibilitada la comprensión del rol fundamental que esta desempeñan en la práctica docente.

De otra parte, las concepciones en sí mismas son objeto de reflexión y estudio en la medida en que constituyen objeto de análisis del profesor pues permiten generar conciencia con referencia a sus prácticas de enseñanza y las concepciones que las alientan, reconociendo así, como desde la diversidad de concepciones que presentan los profesores estos orientan su trabajo pedagógico (Navarro, 2007).Tomando como punto de referencia el eje curricular de Diversidad y teniendo como marco de reflexión el Núcleo Integrador de Problema (NIP) ¿Existe la diversidad? ¿Cuáles son sus significados? Se ha propuesto el presente proyecto. 
Para comprender las diferentes concepciones de los profesores en torno a la experimentación junto con la estructura del trabajo se hizo necesaria una contextualización a partir de la cual se reconocieron aspectos de la localidad, el colegio y el grupo particular de personas afectadas por el desarrollo del trabajo.

Con referencia a la experimentación, esta se asume como una estrategia que permite el acercamiento con la realidad, la cual no solo se limita "al nivel de laboratorio y, por supuesto, no solo es realizar múltiples observaciones (...) pues en ocasiones se experimenta también durante el estudio de la génesis del problema, (...) y siempre implica actividad mental, manipulación mental de la información. Además, la experimentación puede adoptar muchas formas en función del tipo de problema a investigar" (Navarro, 2007 p.52). Es así como, el empalme con la realidad debe hacerse a través de la actividad del niño: manipulativas, sensorial, motriz e intelectual, que implemente objetos y escenarios reales en vez de representaciones o imágenes. (Ministerio de Educación. España, 2001 p. 27). La actividad experimental debe ser parte esencial en la actividad del estudiante. (Ministerio de Educación. España, 2001 p. 27)

Una posible forma de hacer la experimentación, dentro del proceso de enseñanza aprendizaje, es plantear a los alumnos los temas de trabajo en formas de problemas abiertos.

Según Barberá y Valdés (1996) citando a Woolnough y Allsop (1985), establecen que el trabajo practico dentro de la enseñanza se enmarca a partir de tres objetivos, el primero de ellos hace mención a los ejercicios empleados para el desarrollo de destrezas y técnicas prácticas, en segundo lugar, las investigaciones como posibilidad para que los estudiantes se enfrenten a tareas abiertas y finalmente las experiencias por medio de las cuales los estudiantes asumen conciencia frente a los fenómenos naturales. En consecuencia, el valor de las practicas estriba en la posibilidad de desarrollar actitudes cognitivas que implican en igual sentido conocimientos de tipo procedimental y actitudinal (Gil, 1991). Sin embargo el trabajo practico, en especial, desde la enseñanza de las ciencias se asume como un recetario, se convierte en el medio para confirmar teorías y hechos a través de resultados correctos (Barberá y Valdés 1996).

De otro lado, las actividades prácticas aproximan a la consolidación de experiencias que permiten la ampliación de la comprensión en relación con los sucesos y eventos naturales, además permiten contrastar y propiciar el desarrollo del razonamiento practico definido como un "(...) comportamiento inherentemente social e interpretativo propio de la condición humana y necesario para la praxis, un tipo de actividad en la que el desarrollo progresivo del entendimiento del propósito que se persigue emerge durante el ejercicio de la propia actividad" ( Barberá y Valdés 1996). En cuanto a las concepciones en el aula, se entiende por concepción "al estado cognoscitivo corriente (...) con respecto a algún concepto, que incluye su punto de vista, las representaciones y los modos de tratamiento que le son asociados" (Pardo, 2002 p.13)

Encontramos así, que las concepciones del profesor "moldean las estrategias didácticas que se implementan en el aula así como los contenidos que se desarrollan" (Gutiérrez,

Desde el enfoque de Carvajal citado por Gutiérrez en su libro Biología: la teoría de la evolución en la escuela se señala que "el escenario escolar, establece ciertas restricciones o da autonomía a los maestros, lo que conlleva a preguntarse si en realidad los profesores cambian sus ideales epistemológicas y didácticas en el contexto que se desenvuelven a diario, lo cual demostraría las falencias profesionales del campo educativo". Con referencia al tipo de concepciones del docente estas se ven involucradas con las ideas "sobre la ciencia y la forma de aprenderla y enseñarla 
que provienen de la experiencia escolar. Esta estructura producto de la ubicación en el contexto social y de los años de escolaridad se van fundamentando gradualmente." (Gutiérrez, 2009, p. 113)

De acuerdo a investigaciones citadas por Gutiérrez (2009, p 113) este afirma que las concepciones de los docentes se pueden explicitar en los siguientes puntos:

- "Los maestros de ciencias poseen concepciones sobre la naturaleza de la ciencia sea cual sea el instrumento utilizado para su evaluación que no pueden asociarse con alguna posición filosófica de modo excluyente"

- "Las concepciones sobre cómo y que debe aprender el estudiante influyen sobre la puesta en práctica del currículum y están basadas principalmente, en el propio estilo de aprendizaje del maestro"

- "Los docentes en formación tienen confianza en su habilidad para enseñar y una apreciación de la relación establecida en el proceso de enseñanza y de aprendizaje"

- En este trabajo se asume al docente como un sujeto reflexivo en la medida en que es dinámico y estratégico desde su conocimiento teórico y pedagógico por lo que el rol que asume es de mediador entre el encuentro del estudiante con el conocimiento.

\section{Metodología}

La presente investigación se desarrollo bajo el modelo cualitativo que según Taylor y Bogdan (1987; P153) citando a (Geetz, 1983) en su texto mencionan que este enfoque "...proporciona una "una descripción intima" de la vida social de las personas las descripciones intimas presentan detalladamente el contexto y lo significados de los acontecimientos y escenas importantes para los involucrados." (Ver Cuadro 2) Con lo mencionado anteriormente la investigación cualitativa nos posibilitó hacer registros narrativos o escritos de las diferentes maneras en como los profesores entrevistados conciben la experimentación dentro o fuera del aula de clase, además de que nos ayudó a identificar la naturaleza profunda de las realidades, estructuras y dinámicas que viven actualmente algunos maestros a la hora de impartir la cátedra que se les fue asignada dentro del sistema educativo colombiano. No debemos dejar de lado que al hacer este tipo de investigación los resultados obtenidos son de validez interna, es decir para cada uno de los sujetos entrevistados, pero son débiles en validez externa, es decir que no es generalizable para la mayoría de los profesores colombianos los resultados que se obtuvieron en cada una de las entrevistas.

\subsection{Fase de investigación: Contextualización.}

Para entender la estructura del trabajo realizado en el Instituto Pedagógico Nacional, es consiguiente se presenta una breve descripción en la que se abarcan dichos aspectos, entre ellos la localidad de Usaquén, el colegio donde se llevo el proyecto semestral, además del reconocimiento de los docentes de los grados 6 y 7 y los espacios del mismo en los que se llevó a cabo el desarrollo del presente proyecto.

Particularmente en el primer momento de la investigación se realizó la contextualización de la localidad de Usaquén la cual arrojo que es una zona ubicada al norte del distrito capital limitando al norte con la calle 240 , al sur con la 100, al este con los cerros orientales y al oeste con la autopista norte, tal localidad se encuentra dividida en 9 unidades de planeamiento zonal (upz) que se encuentran subdivididas en barios, caracterizándose por ser una zona altamente productiva con presencia de 
centros comerciales, restaurantes, complejos empresariales, entre otros. (Secretaria de Hacienda de Bogotá D.C)

De acuerdo a aspectos ambientales esta zona se encuentra configurada por una zona baja o plana, una zona montañosa con formación de rocas arenosas y "numerosas escorrentías y quebradas como Santa Ana, El contador, San Cristóbal. Además de poseer áreas protegidas que corresponden a los cerros orientales; siendo más de la mitad suelo urbano". (Secretaria de Hacienda de Bogotá D.C)

Por otro lado el IPN es una institución de carácter distrital ubicada en la calle 127 No. 11-20 en la localidad de Usaquén, Bogotá que tuvo apertura según decreto 145 de enero 28 de 1927. El Instituto Pedagógico Nacional (IPN) se considera una "unidad académica y administrativa dependiente de la UPN, la cual busca desarrollar programas de innovación y experimentación educativa" (Agenda IPN). Además de ser un espacio de investigación y práctica docente liderando "procesos de educación formal, educación especial y educación para el trabajo y desarrollo humano de niños, niñas, adolescentes y adultos para la formación de ciudadanos con valores éticos y estéticos desde una perspectiva interdisciplinar que favorezca la construcción de una sociedad democrática y pluralista". (P.E.I del Instituto Pedagógico Nacional en: Universidad Pedagógica Nacional.) Ver cuadro 1.

Con referencia a la población de docentes, estos representan un total de 24 distribuidos en diferentes áreas como matemáticas, historia, geografía, biología, religión, ingles, educación física, entre otras.

\subsection{Fase de recolección de información.}

En el segundo momento de la investigación ya teniendo estructurada la temática a abordar, se enfoco el proyecto con los profesores de los grados 6 y 7 , puesto que el tema está centrado en caracterizar de las diferentes maneras de concebir la experimentación entre ellos, por lo tanto se trabajo a partir de un enfoque cualitativo, utilizando como técnica la entrevista semiestructurada "en la que, como su propio nombre indica, el entrevistador despliega una estrategia mixta, alternando preguntas estructuradas y con preguntas espontáneas .Esto forma es más completa ya que, mientras que la parte preparada permite comparar entre los diferentes candidatos, la parte libre permite profundizar en las características especificas del candidato. Por ello, permite una mayor libertad y flexibilidad en la obtención de información." (Universidad Nacional de Colombia), como instrumento para el registro de los resultados se opto por el diario de campo El desarrollo del presente trabajo implico también, realizar una observación no participante con el profesor y los estudiantes del plantel educativo para poder realizar un análisis en como el profesor designado para aquella clase aplica la experimentación para el desarrollo de la misma.

Para la tabulación de la información recolectada se utilizo la técnica del análisis de contenido teniendo en cuenta que esta se refiere "al conjunto de procedimientos interpretativos de productos comunicativos (mensajes, textos o discursos) que proceden de procesos singulares de comunicación previamente registrados, y que, basados en técnicas de medida, a veces cuantitativas (estadísticas basadas en el recuento de unidades), a veces cualitativas (lógicas basadas en la combinación de categorías) tienen por objeto elaborar y procesar datos relevantes sobre las condiciones mismas en que se han producido aquellos textos, o sobre las condiciones que puedan darse para su empleo posterior." (Piñuel: 2002; P 2) Ver cuadro 2.

Dentro de la tabulación de las respuestas, en primer lugar se utilizaron las categorías de análisis dentro de la cual se enmarca la finalidad de cada pregunta realizada al 
profesor entrevistado, en segundo lugar se empleó la unidad de información donde esta hace referencia a la respuesta casi textual de los profesores entrevistados, enseguida la proposición que se refiere a la extracción de las palabras claves de cada unidad de información y por último las agrupaciones que son una reflexión de la información obtenida en cada pregunta elaborada.

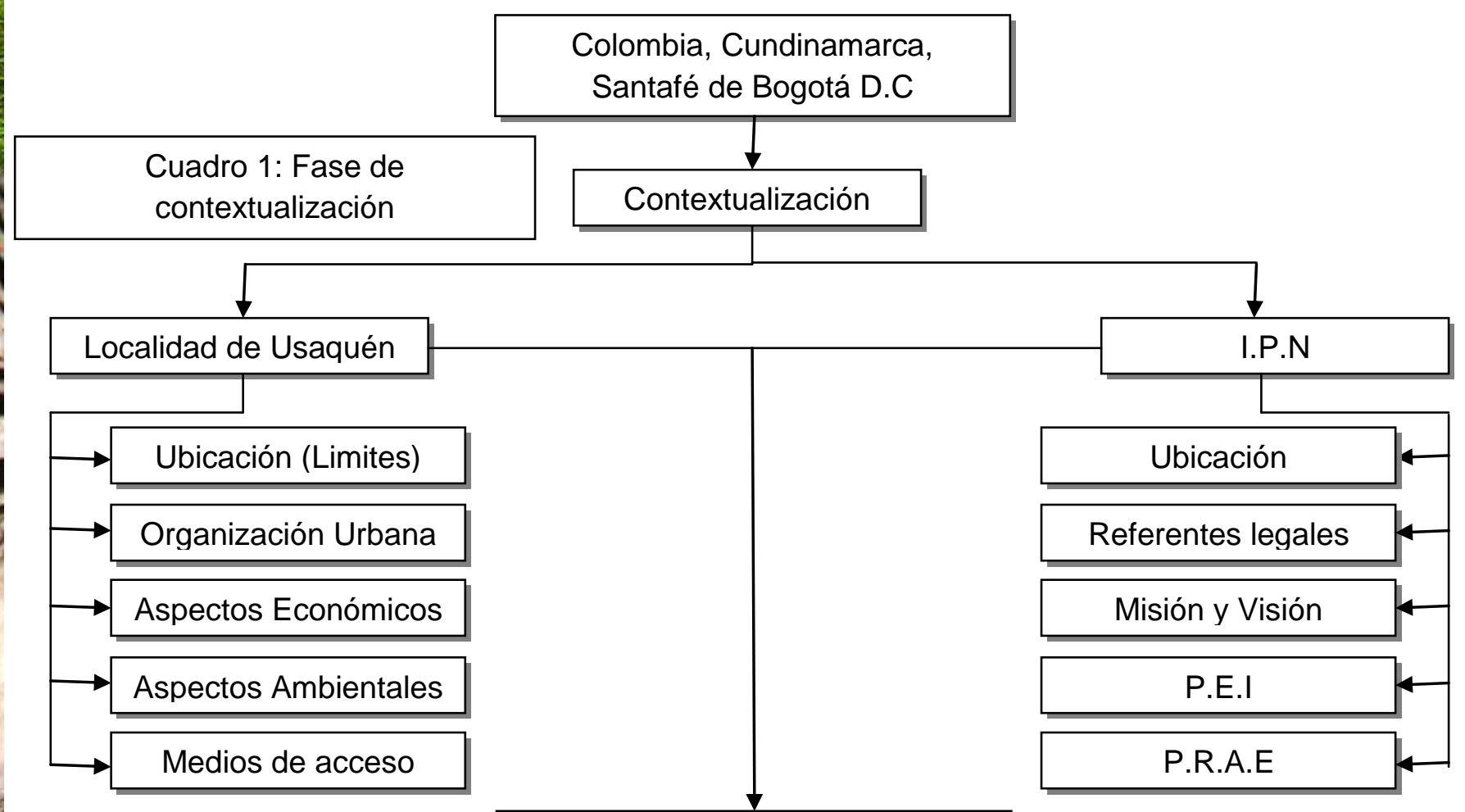

Ubicarse en el contexto de la práctica educativa buscando un primer acercamiento a la comprensión de la diversidad. 


\subsection{Fase de resultados.}

En la tercera fase de trabajo se realizó el análisis de resultados basándonos en lo que nos arrojo la tabulación de la información utilizando como técnica el análisis del contenido.

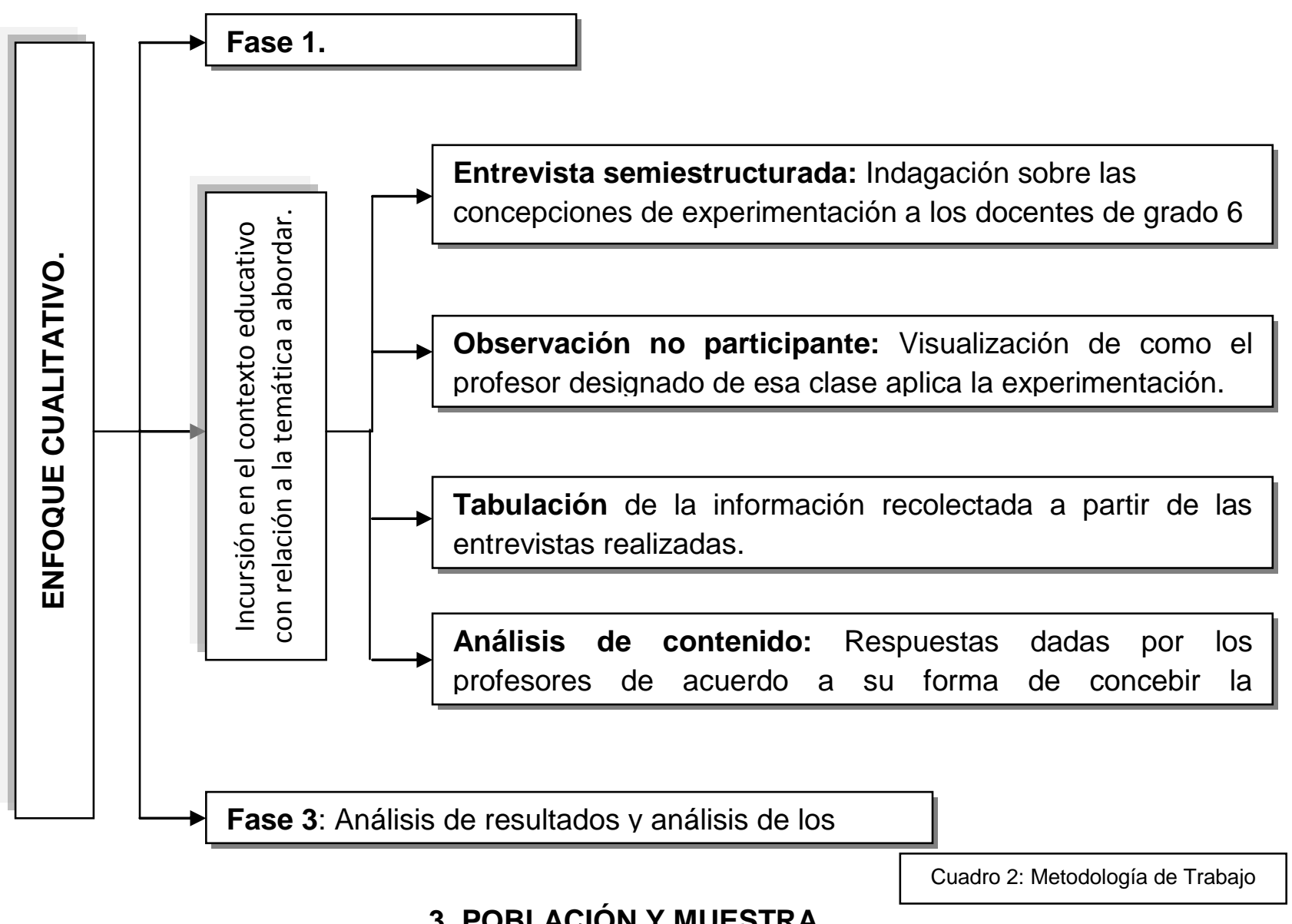

\section{POBLACIÓN Y MUESTRA}

La Población afectada por la investigación fueron los Profesores y estudiantes del IPN. La muestra correspondió a profesores de los grados 6 y 7.

El grupo de profesores de los grados 6 y 7 está comprendido en un total de 24, dentro del desarrollo del presente proyecto se seleccionaron 8 profesores encargados de los espacios académicos de historia, geografía, biología, inglés, lengua castellana y educación física, con lo cual buscábamos identificar la mirada de la experimentación desde diferentes prácticas de enseñanza.

Cabe resaltar que en las tablas y en los análisis de resultados durante el desarrollo de la investigación algunos de los profesores nos solicitaron que sus nombres no fueran revelados, por consiguiente los profesores entrevistados se identifican en el artículo por medio de un número en la escala de 1 a 8.

\section{Resultados y discusión:}

De acuerdo con los resultados obtenidos (Anexo 1) a partir de la entrevista semiestructurada aplicada a los docentes de grado sexto y séptimo del Instituto Pedagógico Nacional, con su respectiva sistematización, mediante la epistemología del análisis de contenido junto con las observaciones no participantes se puede 
señalar en relación con las concepciones acerca de la experimentación, que esta, se concibe en primer lugar como el encuentro de los estudiantes con la experiencia física, los trabajos manuales, los juegos didácticos, que le posibiliten al estudiante consecuencia, se asume la experimentación como estrategia que posibilita el acercamiento con la realidad, que como se obtuvo no solo se da a partir de prácticas de laboratorio sino por el contrario trasciende aún más, , en tanto revisten una manera de acercamiento en la comprensión de la realidad.

Sin embargo, como lo señala un profesor: Mostrando y practicando, desde la practica-error. Es posible analizar el modo como desde está concepción se evidencia la mirada de la experimentación como el trabajo practico para la confirmación a través de resultados correctos (Barberá y Valdés; 1996) y no como posibilidad para la explicación y apropiación del aprendizaje. A partir de lo expuesto, se evidencia que las concepciones de los profesores en relación con la experimentación, delimitan sus prácticas con los otros a través de la enseñanza.

De otro lado, la experimentación, desde lo encontrado también se ve delimitada por los recursos que ofrece la institución, pero no se restringe unilateralmente a estos, sino por el contrario como lo afirman los mismos profesores "Hay pocos recursos, pero de ahí se desprende la creatividad del docente, por buscar elementos del entorno para un aprendizaje". En consecuencia, la experimentación se convierte en iniciativa del profesor y por tanto de las prácticas de enseñanza que este establece.

En relación con las estrategias implementadas por los profesores estas varían de acuerdo con las propias concepciones de los profesores en tanto como lo señala Gutiérrez (2009, p. 113) "Las concepciones sobre cómo y que debe aprender el estudiante influyen sobre la puesta en práctica del currículum y están basadas principalmente, en el propio estilo de aprendizaje del maestro" de este modo, por ejemplo:

El P3 (Profesor 3): señala que la experimentación la vincula con "Actividades relevantes para los estudiantes, sobre hechos reales, situaciones, trabajo individual o grupal, guías, talleres." y de acuerdo a las estrategias que implementa se evidencia nuevamente la importancia que denomina "contexto vivencial", en consecuencia es posible señalar con relación a las estrategias de enseñanza que estas reflejan la puesta en marcha de las propias concepciones del profesor. Adicionalmente, los profesores señalan actividades prácticas, manuales, teoría, brindando importancia a la experiencia, hasta la utilidad de lo aprendido para la vida cotidiana.

Los propósitos principales de los docentes, son formar personas propositivas, responsables, que reflexionen sobre su realidad como sujetos inmersos en una sociedad, personas investigadoras, consientes, sociables, que implementen todo lo que saben en su diario vivir, tomando lo que les sirve y lo que no les sirve. Desde lo anterior, se puede indicar la manera en que la experimentación trasciende tanto el laboratorio como la clase de ciencias e implica entonces una posibilidad de apertura a la comprensión por parte del estudiante.

De esta manera vemos también como la experimentación no se encuentra limitada a los profesores del área de ciencias sino que esta puede adoptar muchas formas en función del tipo de problema a investigar (Navarro, 2007 p. 52) por lo que se puede experimentar desde el planteamiento de los temas en forma de problemas abiertos.

Sin embargo de acuerdo a los análisis desarrollados podemos decir que las estrategias y la manera cómo concibe el profesor la experimentación se encuentra vinculada desde las representaciones que tiene este sobre lo que enseña y como lo 
enseña, por lo que dichas representaciones inciden en el tipo de aprendizaje que se les posibilita a los estudiantes desde el abordaje dado en el aula por lo que la experimentación puede ser usada como una estrategia para la enseñanza de un saber particular.

\section{AGRADECIMIENTOS}

Un especial agradecimiento a todos los profesores del eje curricular diversidad por cada una de las observaciones y orientaciones que hicieron posible hoy la presentación de este trabajo.

Agradecemos a los profesores de los grados 6 y 7 por su disposición ya amabilidad por estar dispuestos a acceder a nuestras preguntas y a la coordinadora del IPN. Finalmente al IPN por abrirnos las puertas para el desarrollo del presente trabajo.

Por último agradecemos al comité evaluador y directivos de la Revista Bio-grafía por hacer posible que nuestro trabajo semestral transcendiera a esferas más grandes además de hacer realidad uno de nuestros sueños como maestros en formación de Licenciatura en Biología.

\section{BIBLIOGRAFÍA.}

- AlZate, M. (2003) La infancia, concepciones y perspectivas. Pereira, Risaralda (Colombia). Ed Papiro.

- BARBERA, O y VALDEZ, P. (1996) El trabajo practico en la enseñanza de las ciencias: Una revisión. Revista de las ciencias, Págs. 365 - 369. Extraído el día 25 de Octubre de 2011 en http://www.raco.cat/index.php/ensenanza/article/viewFile/21466/93439

- BRIONES, G. La investigación en el aula y en la escuela. Ed Guadalupe. Bogotá 1996.

- CANDANEDO, M. (1999) Material pedagógico para la enseñanza de las ciencias en la educación preescolar. Panamá. Editorial universitaria "Carlos Manuel Gasteazoro.

- CERDA, H (1991) Los elementos de la Investigación Como reconocerlos, diseñarlos y construirlos. Editorial El Búho. Bogotá D.C, Colombia.

- ERAZO, M. (1999) El pensamiento del profesor. Universidad Pedagógica Nacional. Bogotá (Colombia).

- GIL, D. (1991) ¿Qué hemos de saber y saber hacer los profesores de ciencias? Intento de síntesis de las aportaciones de la investigación didáctica. Revista Enseñanza de las ciencias. Págs. 96-77. Extraído el 25 de Octubre del 2011 de http://www.cneq.unam.mx/cursos diplomados/diplomados/anteriores/medio su perior/qr/03 mat/construc/que hemos de saber.pdf

- GUtierReZ, A. 2009. Biología la teoría de la evolución en la escuela. Buenos Aires (Argentina). Ed Biblos.

- Proyecto Educativo Institucional IPN. Recuperado el día 25 de Octubre de 2010 en http://www.pedagogica.edu.co/vercontenido. 
- MINISTERIO DE EDUCACIÓN NACIONAL (MEN). (1999). Rol e imagen del maestro.

- MINISTERIO DE EDUCACIÓN. ESPAÑA. (2001) Aulas de verano la experimentación de la enseñanza de las ciencias. Madrid (España). Ed sociedad autónoma de fotocomposición.

- NAVARRO, L. (2007) Autoeficacia del profesor universitario: Eficacia percibida y práctica docente. Ed. Narcea. Pág. 52.

- PIAGET, J. (1981) Psicología y pedagogía. Ariel.

- PIÑUEL. J. (2002) Epistemología, metodología y técnicas del análisis de contenido.

- Programa de iniciativas universitarias para la paz y la convivencia. Universidad Nacional de Colombia. (2011) Recuperado el día 28 de Noviembre de 2010 en: www.piupc.unal.edu.co.

- TAYLOR, J. BOGDAN, R. (1983) Introducción a los métodos de investigación cualitativos de investigación. España. Ed Pardos.

- TAYLOR, J. BOGDAN, R. (1987) Introducción a los métodos de investigación cualitativos de investigación, Edición en Castellano. España. Ed Pardos.

- RIST, R 1997 en: TAYLOR J, BODGAN, R. Introducción a los métodos de investigación. Ed Pardos. España 1984.

- SECRETARIA DE hACIENDA DE BOGOTÁ D.C (2004) Recorriendo Usaquén. Diagnostico físico y socioeconómico de las localidades de Bogotá D.C. Departamento de Planeación Distrital. Bogotá, Colombia.

- ODIEL, M Y POZUELOS, F. Investigando sobre las ideas del alumnado. una experiencia colaborativa en el marco del proyecto escuelas que investigan. Tomado el 23 de octubre de 2010. en: http://www.uhu.es/francisco.pozuelos/biblioteca/ideas alumnado kiki.pdf 


\section{ANEXO 01}

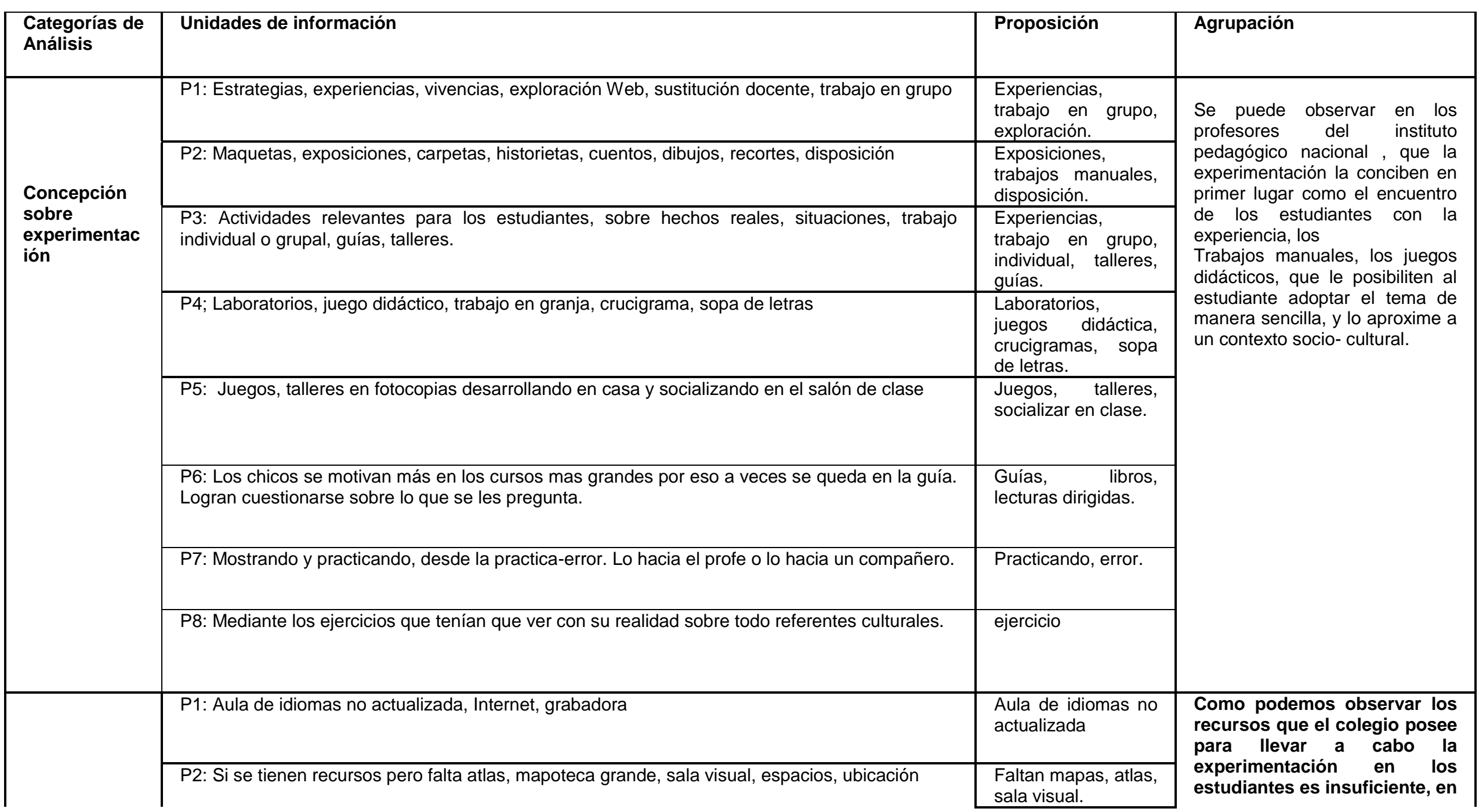


Experimentac

ión en la Institución

\begin{tabular}{|c|c|}
\hline $\begin{array}{l}\text { P3: Hay pocos recursos, pero de ahí se desprende la creatividad del docente, por buscar } \\
\text { elementos del entorno para u aprendizaje. }\end{array}$ & $\begin{array}{l}\text { Pocos recursos, } \\
\text { pero se desprende } \\
\text { la creatividad del } \\
\text { docente. }\end{array}$ \\
\hline $\begin{array}{l}\text { P4; Si, aunque se deben actualizar materiales y equipos de laboratorio, microscopios, equipos } \\
\text { de computo. }\end{array}$ & $\begin{array}{lr}\text { Falta actualizar } & \text { los } \\
\text { materiales } & \text { y } \\
\text { equipos } & \text { de } \\
\text { laboratorio, } & \\
\text { computación } & \\
\end{array}$ \\
\hline $\begin{array}{l}\text { P5: El colegio no cuenta con las reservas suficientes, pero con cada actividad que se realiza se } \\
\text { trata de fomentar la recursividad y potencializar el aprendizaje. }\end{array}$ & $\begin{array}{l}\text { No cuenta con las } \\
\text { reservas } \\
\text { suficientes, pero se } \\
\text { busca } \\
\text { recursividad }\end{array}$ \\
\hline $\begin{array}{l}\text { P6: Guías sí, pero en lo tecnológico esta en deuda, ya no hay sobre todo video beam, con los } \\
\text { de once solo pude } 1 \text { vez. }\end{array}$ & $\begin{array}{l}\text { Lo tecnológico no, } \\
\text { falta video beam. }\end{array}$ \\
\hline \multicolumn{2}{|l|}{$\begin{array}{l}\text { P7: Si, aunque existen momentos en que salimos todos los docentes y hay que adecuarnos al } \\
\text { trabajo. Pues somos } 13 \text { docentes del área. }\end{array}$} \\
\hline $\begin{array}{l}\text { P8: Si me da recursos, antiguos no están actualizados, datan de } 15 \text { a } 18 \text { años no ayuda para el } \\
\text { desarrollo de la clase }\end{array}$ & $\begin{array}{l}\text { Antigüedad de los } \\
\text { recursos }\end{array}$ \\
\hline
\end{tabular}

cualquier área de estudio que se propenda en la institución, puesto que los profesores encuestados, manifiestan inconformidad por 
Bio-grafia: Escritos sobre la Biologia y su Enseñanza Vol. 4 No 7. ISSN 2027-1034.

\begin{tabular}{|c|c|c|c|}
\hline \multirow[b]{3}{*}{$\begin{array}{l}E \\
s \\
t\end{array}$} & P1: Lluvia de ideas, conocimiento previo, apoyo con textos & Ideas, conocimientos previos & \multirow{9}{*}{$\begin{array}{l}\text { Las } \\
\text { estrategias se } \\
\text { basan en } \\
\text { actividades } \\
\text { prácticas, } \\
\text { manuales, } \\
\text { teoría, como } \\
\text { lo podemos } \\
\text { observar en } \\
\text { las } \\
\text { respuestas de } \\
\text { los } \\
\text { profesores } \\
\text { encuestados. }\end{array}$} \\
\hline & P2: Elaboración de atlas, maquetas, frisos, exposiciones, noticias & $\begin{array}{l}\text { Exposiciones, trabajo manual, } \\
\text { noticias }\end{array}$ & \\
\hline & $\begin{array}{l}\text { P3: En cuanto a su metodología varia de acuerdo a su enfoque literario, o gramatical, todo se enfoca } \\
\text { en un contexto vivencial. }\end{array}$ & $\begin{array}{l}\text { Todo va enfocado a la vivencia, y } \\
\text { varia según el enfoque }\end{array}$ & \\
\hline \multirow{3}{*}{$\begin{array}{l}r \\
a \\
t \\
e \\
g \\
i \\
a \\
s\end{array}$} & P4; Guías explicación teórica, y practica, trabajo en granja, y protocolos de laboratorio. & Guías, teoría, practica, protocolos & \\
\hline & P5: Guías, talleres, explicación teórica & Guías, talleres, teoría & \\
\hline & $\begin{array}{l}\text { P6: Presentación del tema, recortes que permitan ver la época, libro de Revolución Industrial, lectura } \\
\text { dirigida y algunos mapas conceptuales, imágenes, cuentos, escritos, narrativos }\end{array}$ & Libro, trabajo manual, escritos. & \\
\hline $\begin{array}{l}\mathbf{d} \\
\mathbf{e}\end{array}$ & P7: Juegos - entrenamientos de intervalo y en circuito & Juegos, entrenamiento. & \\
\hline $\begin{array}{l}e \\
n \\
s \\
e \\
\tilde{n} \\
a \\
n \\
z \\
a\end{array}$ & $\begin{array}{l}\text { P8:Uso de actividades de producción oral y escrita Elaboración de escritos sobre la autobiografía, } \\
\text { ejercicios de expresión oral en clase }\end{array}$ & $\begin{array}{l}\text { Producción } \\
\text { autobiografía }\end{array}$ & \\
\hline \multirow[b]{3}{*}{$\begin{array}{l}P \\
r \\
o \\
p \\
o ́ \\
s\end{array}$} & P1: Comunicación satisfactoria en clase, herramientas de la cotidianidad. & Buena comunicación, & \\
\hline & $\begin{array}{l}\text { P2: Estudiantes propositivos, que no se queden con una sola interpretación, responsables, análisis mas } \\
\text { profundo. }\end{array}$ & $\begin{array}{l}\text { Estudiantes propositivos, } \\
\text { responsables, analíticos. }\end{array}$ & \multirow{2}{*}{$\begin{array}{l}\text { Como } \\
\text { podemos } \\
\text { observar en la } \\
\text { fabulación de } \\
\text { los resultados } \\
\text { obtenidos por } \\
\text { las encuestas, } \\
\text { los } \\
\text { profesores } \\
\text { buscaban, }\end{array}$} \\
\hline & $\begin{array}{l}\text { P3: Participación, conocimiento a través de evidencias, avances, utilidad para su vida, creatividad } \\
\text { innovando nuevos aprendizajes }\end{array}$ & $\begin{array}{l}\text { Participación, conocimiento por } \\
\text { evidencias, utilidad para su vida }\end{array}$ & \\
\hline
\end{tabular}




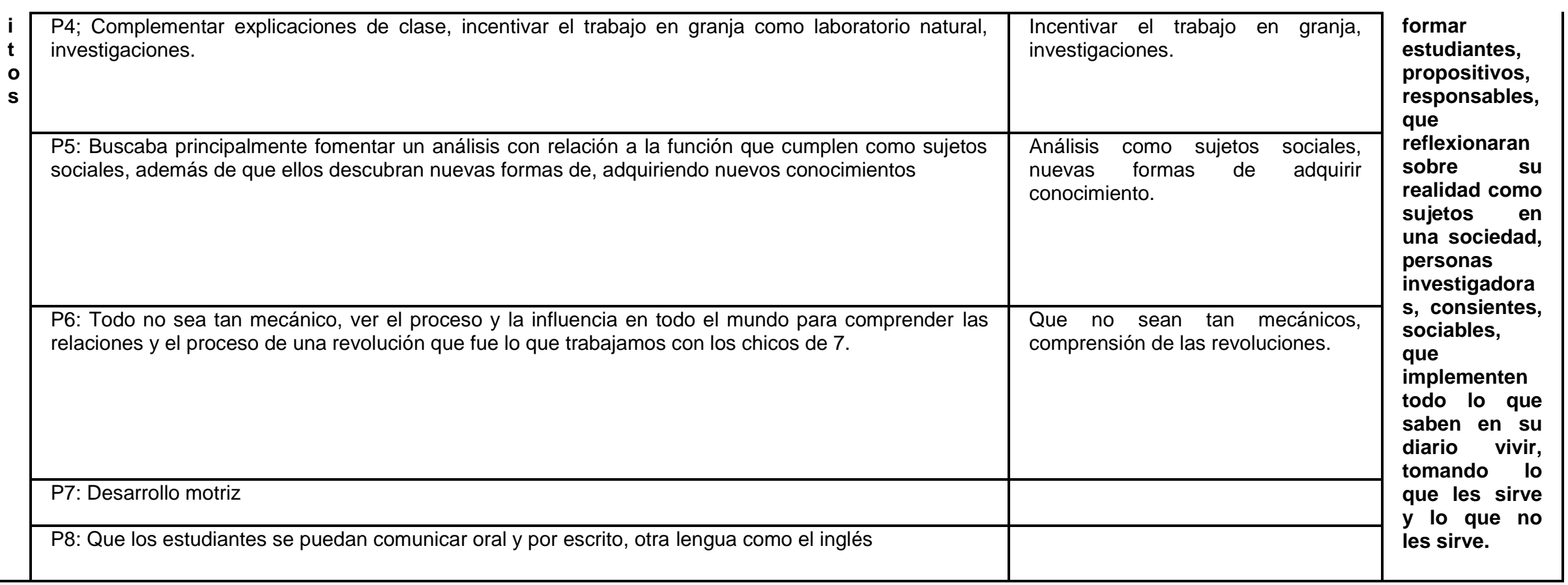

